\title{
On the limiting distribution of a generalized divisor problem for the case $-1 / 2<a<0$
}

\author{
by \\ YUK-KAM LAU (Nancy)
}

1. Introduction. Let $\sigma_{a}(n)=\sum_{d \mid n} d^{a}$. Define

$$
\Delta_{a}(x)=\sum_{n \leq x}^{\prime} \sigma_{a}(n)-\zeta(1-a) x-\frac{\zeta(1+a)}{1+a} x^{1+a}+\frac{1}{2} \zeta(-a)
$$

where $\sum_{n \leq x}^{\prime}$ means that the last term is halved when $x$ is an integer. Taking $a \rightarrow 0^{-}$, we recover the classical error term of Dirichlet's divisor problem

$$
\Delta(x)=\sum_{n \leq x}^{\prime} d(n)-x(\log x+2 \gamma-1)-1 / 4
$$

with $d(n)=\sigma_{0}(n)$. The determination of the precise order of magnitude of $\Delta(x)$ remains an open problem. Nevertheless, there are numerous papers devoted to the study of its properties such as its power moments, $\Omega_{ \pm}$-results, gaps between sign-changes. In particular, Heath-Brown [3] in 1992 showed that $x^{-1 / 4} \Delta(x)$ has a limiting distribution and explored its properties.

Unlike $\Delta(x)$ there are not many results about $\Delta_{a}(x)$. In this paper, we are concerned with the limiting distribution of $\Delta_{a}(x)$ with $-1 / 2<a<0$. It is worthwhile to note that from the available results, $\Delta_{a}(x)$ seems to behave like $\Delta(x)$ only in the range of $-1 / 2<a<0$ (or even $-1 / 2 \leq a<0$ perhaps). When $-1 \leq a<-1 / 2$, the behavior of $\Delta_{a}(x)$ is rather different. Nonetheless the limiting distribution in this case also exists, shown in [7]. A further investigation will be carried out in the sequel paper.

Let us go back to the case $-1 / 2<a<0$. Analogously to the case $a=0$, we can prove that for $-1 / 2<a<0$ and $1 \leq M \leq x$,

$$
\Delta_{a}(x)=\frac{x^{1 / 4+a / 2}}{\pi \sqrt{2}} \sum_{n \leq M} \frac{\sigma_{a}(n)}{n^{3 / 4+a / 2}} \cos (4 \pi \sqrt{n x}-\pi / 4)+O\left(\frac{x^{1 / 2+\varepsilon}}{\sqrt{M}}\right)
$$

2000 Mathematics Subject Classification: Primary 11N60. 
where the $O$-constant depends on $a$ and $\varepsilon$ only. (A proof can be found in [6].) This is the so-called truncated Voronoi formula, which is the basic tool in our discussion.

A direct application of (1.1) and [1, Theorem 4.1] yields the following result.

Theorem 1. For $-1 / 2<a<0, t^{-(1 / 4+a / 2)} \Delta_{a}(t)$ has a limiting distribution $D_{a}(u)$ which is also the distribution of the random series $X=$ $\sum_{n=1}^{\infty} a_{n}\left(t_{n}\right)$ where

$$
a_{n}(t)=\frac{1}{\pi \sqrt{2}} \cdot \frac{\mu(n)^{2}}{n^{3 / 4+a / 2}} \sum_{r=1}^{\infty} \frac{\sigma_{a}\left(n r^{2}\right)}{r^{3 / 2+a}} \cos (2 \pi r t-\pi / 4)
$$

and $t_{1}, t_{2}, \ldots$ are independent random variables uniformly distributed on $[0,1]$. Moreover, $D_{a}(u)=\int_{-\infty}^{u} p_{a}(x) d x$ for some probability density $p_{a}(x)$; $p_{a}(x)$ can be extended to the whole complex plane as an entire function of $x$. Furthermore, for real $x$,

$$
0 \leq p_{a}(x) \ll \exp \left(-|x|^{4 /(1+2|a|)-\varepsilon}\right) .
$$

Define

$$
\text { tail of } D_{a}(u)= \begin{cases}D_{a}(u) & \text { if } u<0, \\ 1-D_{a}(u) & \text { if } u \geq 0 .\end{cases}
$$

In particular, Theorem 1 yields that tail of $D_{a}(u) \ll \exp \left(-|u|^{4 /(1+2|a|)-\varepsilon}\right)$. Our first result is to determine a more precise order of magnitude of $D_{a}(u)$.

Theorem 2. Let $|u| \geq 2$. Then

$$
\exp \left(-c_{1}(a)|u|^{4 /(1+2|a|)}\right) \ll_{a} \text { tail of } D_{a}(u) \ll_{a} \exp \left(-c_{2}(a)|u|^{4 /(1+2|a|)}\right)
$$

where $c_{1}(a)$ and $c_{2}(a)$ are some constants depending on $a$. Also, the implied constants depend on a.

The lower bound is derived by the method in [1, Theorem 5.1] while the upper bound is obtained from the study of its Laplace transform. Such an approach has appeared before, for example, in [2] and [5]. Our proof relies on their underlying principle.

The next result concerns the rate of convergence. The proof follows closely the argument in [7], so we shall give an outline only.

Theorem 3. Define

$$
D_{a, T}(u)=\frac{1}{T} \mu\left\{t \in[1, T]: t^{-(1 / 4+a / 2)} \Delta_{a}(t) \leq u\right\}
$$

where $\mu$ is the Lebesgue measure. Then, for $-1 / 2<a<0$,

$$
D_{a, T}(u)-D_{a}(u) \ll_{a}(\log \log T)^{-(1+2 a) / 8}
$$

where the implied constant depends on a. 
Acknowledgements. The author wishes to thank Prof. D. R. HeathBrown and Dr. Kai-Man Tsang for their valuable comments. Hearty thanks are given to Charlies Tu for unfailing encouragement.

\section{Proof of the lower bound in Theorem 2. Write}

$$
A=\frac{1}{\pi \sqrt{2}} \sum_{r=1}^{\infty} \sigma_{a}\left(r^{2}\right) r^{-(3 / 2+a)} \text {. }
$$

Then we have

$$
\begin{gathered}
\sup _{0 \leq t \leq 1}\left|a_{m}(t)\right| \leq A \sigma_{a}(m) m^{-(3 / 4+a / 2)}, \\
\int_{0}^{1} a_{m}(t) d t=0, \\
\int_{0}^{1} a_{m}(t)^{2} d t=\frac{1}{4 \pi^{2}} \cdot \frac{\mu(m)^{2}}{m^{3 / 2+a}} \sum_{r=1}^{\infty} \frac{\sigma_{a}\left(m r^{2}\right)^{2}}{r^{3+2 a}} .
\end{gathered}
$$

Define $a_{m}^{ \pm}(t)=\max \left(0, \pm a_{m}(t)\right)$. From (2.2) and (2.3), we have

$$
\int_{0}^{1} a_{m}^{+}(t) d t=\int_{0}^{1} a_{m}^{-}(t) d t
$$

and

$$
\int_{0}^{1} a_{m}^{+}(t)^{2} d t+\int_{0}^{1} a_{m}^{-}(t)^{2} d t=\frac{1}{4 \pi^{2}} \cdot \frac{\mu(m)^{2}}{m^{3 / 2+a}} \sum_{r=1}^{\infty} \frac{\sigma_{a}\left(m r^{2}\right)^{2}}{r^{3+2 a}} .
$$

Using (2.1), we obtain

$$
\int_{0}^{1} a_{m}^{ \pm}(t)^{2} d t \leq A \sigma_{a}(m) m^{-(3 / 4+a / 2)} \int_{0}^{1} a_{m}^{+}(t) d t
$$

and hence for any squarefree $m$,

$$
\int_{0}^{1} a_{m}^{+}(t) d t \geq 2 B^{-1} \sigma_{a}(m) m^{-(3 / 4+a / 2)}
$$

where $B=16 \pi^{2} A\left(\sum_{r=1}^{\infty} r^{-(3+2 a)}\right)^{-1}>1$.

Let $n$ be a large integer. For $1 \leq m \leq n$, we define $A_{m}=[0,1]$ if $m$ is non-squarefree, and

$$
A_{m}=\left\{t \in[0,1]: a_{m}(t)>B^{-1} \sigma_{a}(m) m^{-(3 / 4+a / 2)}\right\}
$$

otherwise. For squarefree $m$, it is apparent that

$$
\sup _{0 \leq t \leq 1}\left|a_{m}(t)\right| \mu\left(A_{m}\right)+\frac{1}{B} \cdot \frac{\sigma_{a}(m)}{m^{3 / 4+a / 2}} \mu\left(A_{m}^{\mathrm{c}}\right) \geq \int_{0}^{1} a_{m}^{+}(t) d t .
$$


Hence from (2.1), (2.4) and $\mu\left(A_{m}^{\mathrm{c}}\right) \leq 1$ we get

$$
1 / B^{\prime} \leq \mu\left(A_{m}\right) \leq 1
$$

where $B^{\prime}=A B$. By Markov's inequality, we have

$$
\operatorname{Pr}\left(\left|\sum_{m=n+1}^{\infty} a_{m}\left(t_{m}\right)\right| \leq 2 \sqrt{K}\right) \geq 1-\frac{1}{4 K} \sum_{m=1}^{\infty} \int_{0}^{1} a_{m}(t)^{2} d t \geq \frac{3}{4}
$$

where $\operatorname{Pr}(\#)$ denotes the probability of the event \# and

$$
K=\sum_{m=1}^{\infty} \int_{0}^{1} a_{m}(t)^{2} d t
$$

Define

$$
E_{n}=\left\{\left(t_{1}, t_{2}, \ldots\right): t_{m} \in A_{m} \text { for } 1 \leq m \leq n \text { and }\left|\sum_{m=n+1}^{\infty} a_{m}\left(t_{m}\right)\right| \leq 2 \sqrt{K}\right\} .
$$

Then we get

$$
\operatorname{Pr}\left(E_{n}\right)=\prod_{m=1}^{n} \operatorname{Pr}\left(A_{m}\right) \operatorname{Pr}\left(\left|\sum_{m=n+1}^{\infty} a_{m}\left(t_{m}\right)\right| \leq 2 \sqrt{K}\right) \geq \frac{3}{4} e^{-n \log B^{\prime}}
$$

due to $\operatorname{Pr}\left(A_{m}\right)=\mu\left(A_{m}\right)$ and $(2.5)$. When $\left(t_{1}, t_{2}, \ldots\right) \in E_{n}$,

$$
\sum_{m=1}^{\infty} a_{m}\left(t_{m}\right) \geq \frac{1}{B} \sum_{\substack{m \leq n \\ m \text { squarefree }}} \frac{\sigma_{a}(m)}{m^{3 / 4+a / 2}}-2 \sqrt{K} \gg n^{1 / 4+|a| / 2} .
$$

Here and in what follows, the implied constants may depend on $a$. Replacing $n$ by $\left[u^{4 /(1+2|a|)}\right]$, we obtain $1-D(u) \gg \exp \left(-c_{1}(a) u^{4 /(1+2|a|)}\right)$ for all sufficiently large $u$. The case of $D(-u)$ can be proved in a similar way.

3. Proof of the upper bound in Theorem 2. To prove it, we need the following result which is contained in [5]. For the sake of completeness, we give a proof as well.

A positive measurable function $\phi(x)$ defined for sufficiently large positive $x$ is called a regularly varying function with index $\alpha$ if

$$
\lim _{x \rightarrow \infty} \phi(\lambda x) / \phi(x)=\lambda^{\alpha} \quad \text { for any } \lambda>1 .
$$

$\psi(x)$ is called an asymptotic inverse of $\phi(x)$ if $\lim _{x \rightarrow \infty} \psi(\phi(x)) / x=1$.

Lemma 3.1. Let $X$ be a real random variable with probability distribution $D(x)$, let $\phi(x)$ be a regularly varying function with index $0<\alpha<1$, and let $\psi(x)$ be an asymptotic inverse of $x / \phi(x)$. Suppose that $D(x)>0$ for any $x>0$ and $L \in(0, \infty)$. We have 
(a) if $\lim \sup _{\lambda \rightarrow \infty} \psi(\lambda)^{-1} \log E(\exp (\lambda X)) \leq L$, then

$$
\limsup _{x \rightarrow \infty} \frac{1}{x} \log (1-D(\phi(x))) \leq-\alpha\left(\frac{1-\alpha}{L}\right)^{(1-\alpha) / \alpha},
$$

(b) if $\lim \sup _{\lambda \rightarrow \infty} \psi(\lambda)^{-1} \log E(\exp (-\lambda X)) \leq L$, then

$$
\limsup _{x \rightarrow \infty} \frac{1}{x} \log D(-\phi(x)) \leq-\alpha\left(\frac{1-\alpha}{L}\right)^{(1-\alpha) / \alpha} .
$$

Proof. The proofs of (a) and (b) are similar and we prove part (b) only. Write $A=\limsup _{x \rightarrow \infty} \log D(-\phi(x)) / x \leq 0$; the result is obviously true if $A=-\infty$. Let $\xi>0$ be fixed and $\eta>0$. Then

$$
\begin{aligned}
E\left(\exp \left(-\frac{\eta}{\phi(\eta)} X\right)\right) & =\int_{-\infty}^{\infty} \exp \left(-\frac{\eta}{\phi(\eta)} x\right) d D(x) \\
& \geq \int_{-\infty}^{-\phi(\xi \eta)} \exp \left(-\frac{\eta}{\phi(\eta)} x\right) d D(x) \\
& \geq \exp \left(\eta \frac{\phi(\xi \eta)}{\phi(\eta)}\right) D(-\phi(\xi \eta))
\end{aligned}
$$

Hence,

$$
\frac{1}{\eta} \log E\left(\exp \left(-\frac{\eta}{\phi(\eta)} X\right)\right) \geq \frac{\phi(\xi \eta)}{\phi(\eta)}+\frac{1}{\eta} \log D(-\phi(\xi \eta)) .
$$

Then, for any $\varepsilon \in(0,1)$, there exist infinitely many $\eta \geq \eta_{0}(\varepsilon)$ such that

$$
\frac{1}{\eta} \log E\left(\exp \left(-\frac{\eta}{\phi(\eta)} X\right)\right) \geq \frac{\phi(\xi \eta)}{\phi(\eta)}+(A-\varepsilon) \xi .
$$

Therefore,

$$
\limsup _{\eta \rightarrow \infty} \frac{1}{\eta} \log E\left(\exp \left(-\frac{\eta}{\phi(\eta)} X\right)\right) \geq \xi^{\alpha}+A \xi
$$

Taking $\lambda=\eta / \phi(\eta)$, we have $\lambda \rightarrow \infty$ as $\eta \rightarrow \infty$ since $0<\alpha<1$ (see [8, Section 1.1]), and so

$$
\limsup _{\lambda \rightarrow \infty} \frac{1}{\psi(\lambda)} \log E(\exp (-\lambda X)) \geq \xi^{\alpha}+A \xi .
$$

From the hypothesis in (b), we obtain $L \geq \xi^{\alpha}+A \xi$, which holds for all $\xi>0$. Let us write $A=-H(H>0)$. Then we get $L \geq \xi^{\alpha}-H \xi$ and by taking $\xi=(\alpha / H)^{1 /(1-\alpha)}$, we have

$$
H \geq \alpha\left(\frac{1-\alpha}{L}\right)^{(1-\alpha) / \alpha} .
$$

Our assertion follows. 
As $X=\sum_{n=1}^{\infty} a_{n}\left(t_{n}\right)$ where $t_{n}$ 's are independent random variables uniformly distributed on $[0,1]$, we have

$$
E(\exp ( \pm \lambda X))=\prod_{n=1}^{\infty} E\left(\exp \left( \pm \lambda a_{n}\left(t_{n}\right)\right)\right)=\prod_{n=1}^{\infty} \int_{0}^{1} \exp \left( \pm \lambda a_{n}(t)\right) d t .
$$

Now, we take $\phi(x)=x^{(1+2|a|) / 4}, \psi(x)=x^{4 /(3+2 a)}$ and $N=\left[\lambda^{4 /(3+2 a)}\right]$. We want to give an upper bound for $\log E(\exp ( \pm \lambda X))$ where $\lambda \geq 1$. Therefore, we consider the integrals (inside the product) according to the following three cases.

CASE (i): $n \leq N$. Using $\sigma_{a}\left(n r^{2}\right) \leq \sigma_{a}(n) \sigma_{a}\left(r^{2}\right)$,

$$
\int_{0}^{1} \exp \left( \pm \lambda a_{n}(t)\right) d t \leq \exp \left(\lambda A \frac{\sigma_{a}(n) \mu(n)^{2}}{n^{3 / 4+a / 2}}\right) .
$$

Recall that $A=(\pi \sqrt{2})^{-1} \sum_{r=1}^{\infty} \sigma_{a}\left(r^{2}\right) r^{-(3 / 2+a)}$.

CASE (ii): $n>N$ and $\lambda A \sigma_{a}(n)<n^{3 / 4+a / 2}$. Using the inequality $e^{x} \leq$ $1+x+x^{2}$ for $-\infty<x \leq 1$ and $a_{n}(t) \leq \lambda A \sigma_{a}(n) / n^{3 / 4+a / 2}$, we obtain with (2.2),

$$
\begin{aligned}
\int_{0}^{1} \exp \left( \pm \lambda a_{n}(t)\right) d t & \leq \int_{0}^{1}\left(1+\lambda a_{n}(t)+\lambda^{2} a_{n}(t)^{2}\right) d t \\
& \leq 1+(\lambda A)^{2} \frac{\sigma_{a}(n)^{2} \mu(n)^{2}}{n^{3 / 2+a}} \\
& \leq \exp \left((\lambda A)^{2} \frac{\sigma_{a}(n)^{2} \mu(n)^{2}}{n^{3 / 2+a}}\right)
\end{aligned}
$$

since $e^{x} \geq 1+x$ for all real $x$.

CASE (iii): $n>N$ and $\lambda A \sigma_{a}(n) \geq n^{3 / 4+a / 2}$. As $e^{x} \leq e^{x^{2}}$ for $|x| \geq 1$ or $x=0$,

$$
\int_{0}^{1} \exp \left( \pm \lambda a_{n}(t)\right) d t \leq \exp \left(\lambda A \frac{\sigma_{a}(n) \mu(n)^{2}}{n^{3 / 4+a / 2}}\right) \leq \exp \left((\lambda A)^{2} \frac{\sigma_{a}(n)^{2} \mu(n)^{2}}{n^{3 / 2+a}}\right) .
$$

Since

$$
\begin{aligned}
& \sum_{n \leq x} \sigma_{a}(n)=\zeta(1-a) x+O\left(x^{1+a}\right) \\
& \sum_{n \leq x} \sigma_{a}(n)^{2}=\zeta(1-2 a) \zeta(1-a)^{2} \zeta(1-2 a)^{-1} x+O\left(x^{1+a}\right),
\end{aligned}
$$

we have 


$$
\begin{aligned}
\log E(\exp ( \pm \lambda X)) & \leq \lambda A \sum_{n \leq N} \frac{\sigma_{a}(n) \mu(n)^{2}}{n^{3 / 4+a / 2}}+(\lambda A)^{2} \sum_{n>N} \frac{\sigma_{a}(n)^{2} \mu(n)^{2}}{n^{3 / 2+a}} \\
& \leq c_{1} \lambda A N^{1 / 4+|a| / 2}+c_{2}(\lambda A)^{2} N^{-1 / 2+|a|} \\
& \leq c_{3} \lambda^{4 /(3+2 a)}
\end{aligned}
$$

where $c_{1}, c_{2}$ and $c_{3}$ are some positive constants depending on $a$.

Thus,

$$
\limsup _{\lambda \rightarrow \infty} \frac{1}{\psi(\lambda)} \log E(\exp ( \pm \lambda X)) \leq c_{3} .
$$

Note that $D_{a}(u)>0$ for all $u$ from the lower bound. By Lemma 3.1 and replacing $\phi(x)$ by $u$, i.e. $x=u^{4 /(1+2|a|)}$, our proof is then complete.

4. Proof of Theorem 3. Define $F_{a}(t)=t^{-(1 / 2+a)} \Delta_{a}\left(t^{2}\right)$ and let $a_{n}(t)$ be defined as in (1.2). By taking $M=T^{2}$ in (1.1), we have for $1 \leq N \leq T$,

$$
\begin{aligned}
& \int_{T}^{2 T}\left|F_{a}(t)-\sum_{n \leq N} a_{n}\left(\gamma_{n} t\right)\right|^{2} d t \\
& \ll \int_{T}^{2 T}\left|\sum_{N<n \leq T^{2}}^{\prime} \frac{\sigma_{a}(n)}{n^{3 / 4+a / 2}} \cos (4 \pi \sqrt{n} t-\pi / 4)\right|^{2} d t \\
& \quad+\int_{T}^{2 T}\left|\sum_{n \leq N} \frac{\mu(n)^{2}}{n^{3 / 4+a / 2}} \sum_{r>T / \sqrt{n}} \frac{\sigma_{a}\left(n r^{2}\right)}{r^{3 / 2+a}} \cos (4 \pi r \sqrt{n} t-\pi / 4)\right|^{2} d t+T^{2|a|+\varepsilon}
\end{aligned}
$$

where $\sum^{\prime}$ sums over integers of the form $n=m r^{2}$ where $m>N$ is squarefree. Then the first integral on the right hand side is evaluated as in Ivić [4, Theorem 13.5] while the second one is bounded trivially. We obtain for $1 \leq N \leq T$,

$$
\int_{T}^{2 T}\left|F_{a}(t)-\sum_{n \leq N} a_{n}\left(\gamma_{n} t\right)\right|^{2} d t \ll T N^{|a|-1 / 2}+T^{2|a|+\varepsilon} N^{1+\varepsilon} .
$$

Let us write $D_{F, T}(u)=T^{-1} \mu\left\{t \in[1, T]: F_{a}(t) \leq u\right\}$. Then, applying the argument in $[7,(5.1)]$, we have for any $r>2$,

$$
D_{a, T}(u)-D_{a}(u) \ll \sup _{T^{1 / r} \leq v \leq T^{1 / 2}}\left|D_{F, v}(u)-D_{a}(u)\right|+T^{2 / r-1} .
$$

Thus, we consider $D_{a}(u)-D_{F, T}(u)$ and we have

$$
D_{a}(u)-D_{F, T}(u) \ll \frac{1}{R}+\int_{-R}^{R}\left|\frac{\chi_{a, T}(\alpha)-\chi_{a}(\alpha)}{\alpha}\right| d \alpha
$$


where $\chi_{a, T}(\alpha)$ and $\chi_{a}(\alpha)$ are the characteristic functions of $D_{F, T}$ and $D_{a}$ respectively. Define

$$
\chi_{N, T}(\alpha)=\frac{1}{T} \int_{1}^{T} \prod_{n=1}^{N} e\left(\alpha a_{n}\left(\gamma_{n} t\right)\right) d t \quad \text { and } \quad \chi_{N}(\alpha)=\prod_{n=1}^{N} \int_{0}^{1} e\left(\alpha a_{n}(t)\right) d t .
$$

Taking $N=2[(\log \log T) / 4], R=N^{(1-2|a|) / 8}$ and following [7, (5.3)-(5.4)], we obtain by $(4.1)$,

$$
D_{a}(u)-D_{F, T}(u) \ll \frac{1}{R}+R N^{|a| / 2-1 / 4}+\int_{-R}^{R}\left|\frac{\chi_{N, T}(\alpha)-\chi_{N}(\alpha)}{\alpha}\right| d \alpha .
$$

We follow $[7,(5.5)-(5.7)]$ (with the same choices of $M$ and $\delta$ ) to evaluate $\left|\chi_{N, T}(\alpha)-\chi_{N}(\alpha)\right|$. Then we can get

$$
\left|\chi_{N, T}(\alpha)-\chi_{N}(\alpha)\right| \ll(|\alpha|+1)(\log T)^{-1 / 4+|a| / 2+\varepsilon},
$$

and $\left|\chi_{N, T}(\alpha)-\chi_{N}(\alpha)\right| \ll|\alpha| N^{1 / 4+|a| / 2+\varepsilon}$ if $|\alpha| \leq(\log T)^{-1}$. This yields

$$
\int_{-R}^{R}\left|\frac{\chi_{N, T}(\alpha)-\chi_{N}(\alpha)}{\alpha}\right| d \alpha \ll(\log T)^{-1 / 4+|a| / 2+\varepsilon} .
$$

Together with (4.2) and (4.3), our result follows.

\section{References}

[1] P. M. Bleher, Z. Cheng, F. J. Dyson and J. L. Lebowitz, Distribution of the error term for the number of lattice points inside a shifted circle, Comm. Math. Phys. 154 (1993), 433-469.

[2] T. Hattori and K. Matsumoto, A limit theorem for Bohr-Jessen's probability measures of the Riemann zeta-function, J. Reine Angew. Math. 507 (1999), 219-232.

[3] D. R. Heath-Brown, The distribution and moments of the error term in the Dirichlet divisor problem, Acta Arith. 60 (1992), 389-415.

[4] A. Ivić, The Riemann Zeta-Function, Wiley, New York, 1985.

[5] Y. Kasahara, Tauberian theorems of exponential type, J. Math. Kyoto Univ. 18 (1978), 209-219.

[6] I. Kiuchi, On an exponential sum involving the arithmetic function $\sigma_{a}(n)$, Math. J. Okayama Univ. 29 (1987), 193-205.

[7] Y. K. Lau, On the existence of limiting distributions of some number-theoretic error terms, manuscript.

[8] E. Seneta, Regularly Varying Functions, Lecture Notes in Math. 508, Springer, 1976.

Institut Élie Cartan

Université Henri Poincaré (Nancy 1)

54506 Vandœuvre-lès-Nancy Cedex, France

E-mail: lau@antares.iecn.u-nancy.fr

Received on 25.2.2000

and in revised form on 18.9.2000 